\title{
Primary B-cell lymphoblastic lymphoma of the testis
}

\author{
Flavia Tombolini ${ }^{1}$, Vito Lacetera ${ }^{1}$, Guido Gini ${ }^{2}$, Debora Capelli ${ }^{2}$, Pietro Leoni ${ }^{2}$, Rodolfo Montironi ${ }^{3}$, \\ Andrea Benedetto Galosi ${ }^{4}$, Giovanni Muzzonigro ${ }^{1}$ \\ ${ }^{1}$ Institute of Urology, ${ }^{2}$ Institute of Hematology, ${ }^{3}$ Institute of Pathological Anatomy and Histopathology, AOU United Hospitals, \\ Polytechnic University of Marche Region, Ancona, Italy; \\ ${ }^{4}$ Division of Urology, A. Murri Hospital, Fermo, Italy.
}

\begin{abstract}
Summary We present a rare case of primary lymphoblastic B-cell lymphoma of the testis focusing on ultrasonographic and pathological features and clinical implications. Pathological examination revealed primary testicular lymphoblastic B-cell lymphoma which was treated with adjuvant chemotherapy, including rachicentesis with administration of chemotherapy and with radiotherapy of contralateral testis. Primary testicular lymphoblastic B cell lymphoma is an aggressive disease and it is necessary a multimodal therapy (surgery, chemotherapy and radiotherapy) to prevent metastasis.
\end{abstract}

KEY WORDS: B-cell lymphoblastic lymphoma; Primary testicular lymphoma; Testicular ultrasound.

Submitted 3 October 2014; Accepted 31 October 2014

\section{INTRODUCTION}

Primary testicular lymphoblastic B-cell lymphoma is a rare tumor, it represents $7 \%$ of all testicular cancer $(<2 \%$ in men under 50 years of age) and it accounts for $1 \%$ of all non-Hodgkin's lymphomas. It is the most common bilateral testicular tumor, with 10-18\% cases of synchronous or asynchronous involvement of contralateral testis (1-4). It has no pathognomonic clinical and ultrasonographic features and a diagnosis before surgery is uncommon. It needs specific adjuvant chemotherapy and radiotherapy because of the high risk of metastatic process at skin, central nervous system, contralateral testis and other organs. A multidisciplinary care is the key of treatment.

\section{CAse RePort}

A 39 years old man was referred to our clinic with acute pain at the left testis; a swelling hard mass in the inferior part of the left testicle was palpable; the right testis was normal without any associated symptoms of lower urinary tract infection. After a first clinical evaluation an infective orchiepididymitis was suspected and antibiotics were administered for 2 weeks. He had no comorbidities and no history of traumatic events in the inguinal and scrotal region. He underwent surgical correction of left varicocele 12 years before with a complete resolution of the problem. Scrotal ultrasound showed an hypoechoic area with homogeneous aspects in the inferior part of the left testicle, size 2.7 x $1.3 \mathrm{~cm}$. Scrotal eco-power-Doppler showed high blood perfusion (Figure 1) and increased vascularization of the left epididymis if compared to the right one. Tumoral markers were normal. Patient underwent left orchifunicolectomy and implantation of testicular prosthesis. Intraoperative pathological evaluation described an extended cellular neoplastic population with mitotic aspects, high nucleocytoplasmic ratio and irregular nucleus. Definitive pathological report described a creamy white nodular proliferation of 2,8 x 2,5 cm in lower pole of testis; microscopic examination was positive for lymphoblastic B-cells with intermediate size and irregular nucleus (Figure 2). Immunohistochemical expression pattern was: CD45 positive, CD79a-CD10-CD38 positive, Bcll, Bcl6, CD57, MUM 1 CD99 and CD117 negative; focal areas were CD20, Bcl 2, TdT and CD3 positive (Figure 3). The proliferation index, as detected by MIBl anti-

\section{Figure 1.}

High blood perfusion at colour-Doppler examination in the inferior part of testis.

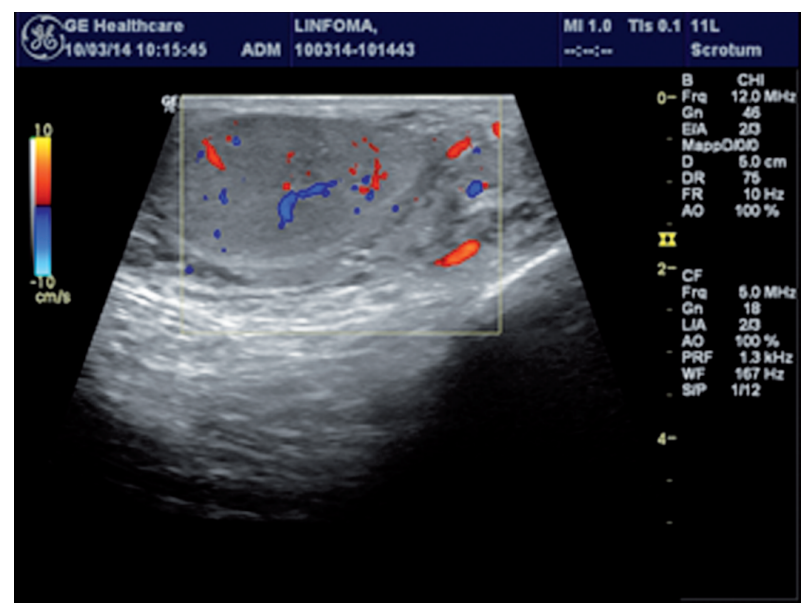

No conflict of interest declared. 


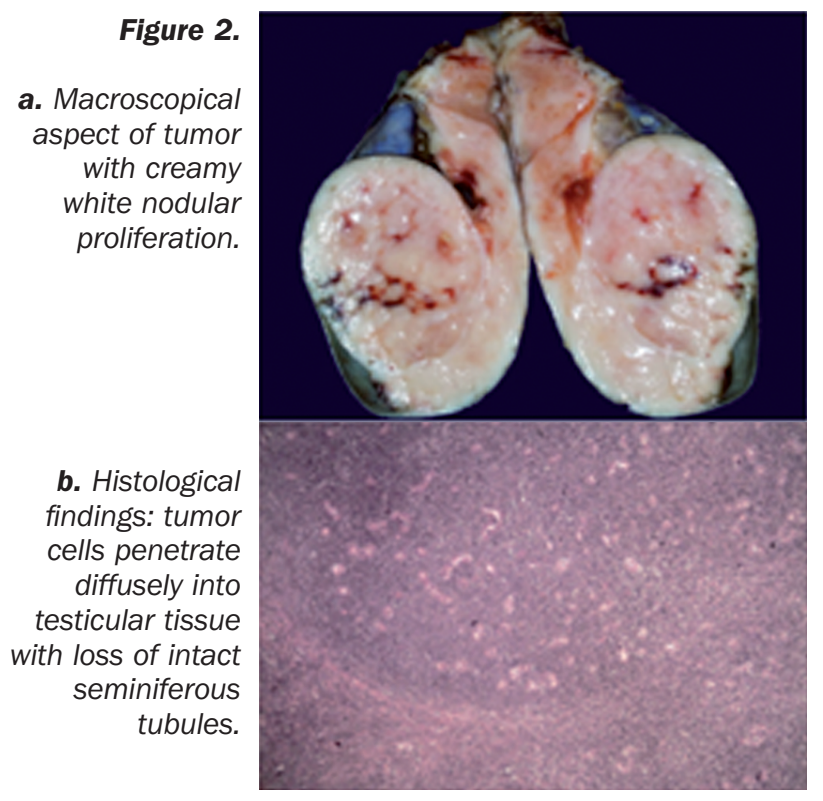

Figure 3. c. Histological
findings: cells
demonstrated
enlarged nucleii
with little
nucleoli and
decrement of
cytoplasm.

d. Hematoxylin and eosin). Immunihistoche mical panel with TdT positive antibodies.

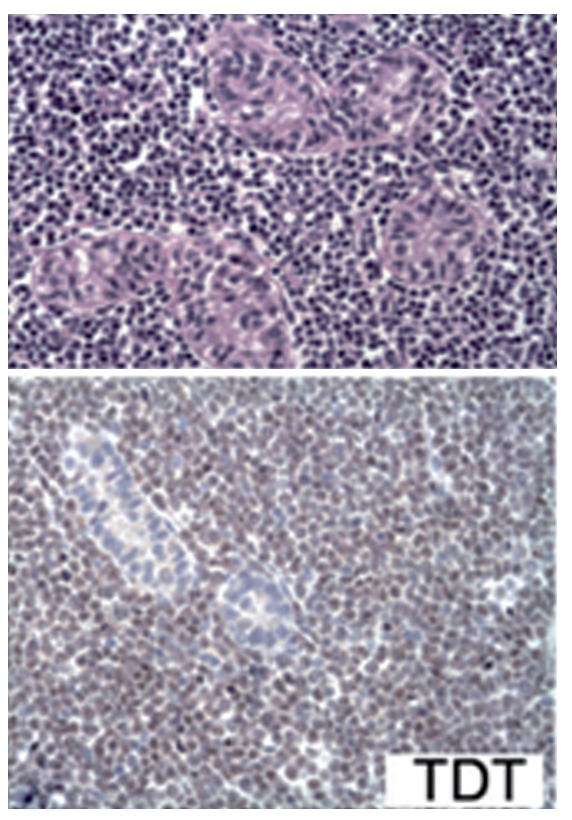

body, was 70\%. This lesion was classified as a primary testicular lymphoblastic B-cell lymphoma with focal expression of CD3, confined to the testicle. Patient underwent total-body computed tomography (CT), which resulted negative for metastasis. A multidisciplinary discussion with urologists, oncologists, radiation oncologists and hematologists was done. After a negative bone marrow biopsy the patient underwent to 6 cycles of hyper-CVAD chemotherapy (cyclophosphamide, vincristine, doxorubicin, and dexamethasone given as course A, followed by methotrexate and cytarabine given as course B), rachicentesis with administration of methotrexate $12 \mathrm{mg}$ and cytarabin 100 mg (2 for each cycle), and radiotherapy of contralateral testis. We proposed also cryoconservation of sperm before starting adjuvant treatment. After 6 months of follow-up the patient is alive and without disease.
Discussion is posted in Supplementary Materials in www.aiua.it

\section{Conclusions}

Primary testicular lymphoma is not associated to pathognomonic findings at ultrasound and is not different from other testis neoplasms. Primary testicular lymphoblastic B lymphoma is an aggressive disease and it is necessary a multimodality therapy (surgery, chemotherapy and radiotherapy) to prevent metastasis.

\section{References}

1. Emura A, Kudo S, et al. Testicular malignant lymphoma: imaging and diagnosis. Radiat Med. 1996; 14:121-126.

2. Mazzu D, Jeffrey RB, et al. Lymphoma and leukemia involving the testicles: findings on grey scale and colour-Doppler sonography. Am J Roentgenol. 1995; 164:645-647.

3. Ahmad SS, Idris SF, et al. Primary testicular lymphoma. Clin Oncol. 2012; 24:358-65.

4. Cheah CY, Wirth A, Seymour JF. Primary testicular lymphoma. Blood. 2014; 123:486-93.

\section{Correspondence}

Flavia Tombolini, MD, Resident in Urology

flavia.tombolini@virgilio.it

Vito Lacetera, MD

vlacetera@gmail.com

Giovanni Muzzonigro, MD

g.muzzonigro@univpm.it

Guido Gini, MD, Hematologist

Guido.Gini@ospedaliriuniti.marche.it

Debora Capelli, MD, Hematologist

Debora.Capelli@ospedaliriuniti.marche.it

Pietro Leoni, MD, Hematologist

p.leoni@univpm.it

Rodolfo Montironi, MD, Pathologist

R.Montironi@univpm.it

AOU United Hospitals Polytechnic University of Marche Region,

Ancona, Italy

Andrea B. Galosi, MD (Corresponding Author)

galosiab@yahoo.it

Division of Urology, Dept. of Surgery, "Augusto Murri” General Hospital Area Vasta 4, Az. Sanitaria Unica Regione Marche

63900 Fermo (FM), Italy 